\title{
COHEN-MACAULAY BINOMIAL EDGE IDEALS OF CACTUS GRAPHS
}

\author{
GIANCARLO RINALDO
}

\begin{abstract}
We classify the Cohen-Macaulay binomial edge ideals of cactus and bicyclic graphs.
\end{abstract}

\section{INTRODUCTION}

In 2010, binomial edge ideals were introduced in [6] and appeared independently also in [8]. Let $S=K\left[x_{1}, \ldots, x_{n}, y_{1}, \ldots, y_{n}\right]$ be the polynomial ring in $2 n$ variables with coefficients in a field $K$. Let $G$ be a graph on vertex set $[n]$ and edges $E(G)$. For each $\{i, j\} \in E(G)$ with $i<j$, we associate a binomial $f_{i j}=x_{i} y_{j}-x_{j} y_{i}$. The ideal $J(G)$ of $S$ generated by $f_{i j}=x_{i} y_{j}-x_{j} y_{i}$ such that $i<j$, is called the binomial edge ideal of $G$. Any ideal generated by a set of 2 -minors of a $2 \times n$-matrix of indeterminates may be viewed as the binomial edge ideal of a graph.

Algebraic properties of binomial edge ideals in terms of properties of the underlying graph were studied in [6], [4] and [9]. In [4] and [9] the authors considered the Cohen-Macaulay property of these graphs. Recently nice results on Cohen-Macaulay bipartite graphs and blocks have been obtained (see [7, 2] and [1]).

However, the classification of Cohen-Macaulay binomial edge ideals in terms of the underlying graphs is still widely open and, as in the case of monomial edge ideals introduced in [11, it seems rather hopeless to give a full classification.

The aim of this paper is to extend the results of [10] where we classify Cohen-Macaulay and unmixed binomial edge ideals $J(G)$ with deviation, namely the difference between the minimum number of the generators and the height of $J(G)$, less than or equal to 1 . This invariant has an interesting combinatorial interpretation: if $J(G)$ is unmixed than its deviation is $|E(G)|-n+c$ where $c$ is the number of connected components of $G$ (see Remark 2.1 of [10]). Hence deviation represents the minimum number of edges that must be removed from the graph to break all its cycles making it into a forest (see Chapter 4 of [5]).

In section 2 we give a classification Cohen-Macaulay and unmixed binomial edge ideals $J(G)$ when $G$ is a cactus graph, a graph whose blocks are cycles. This is a natural extension to the result obtained in [10] and useful to study binomial edge ideals by the deviation invariant. 
In section 3 as an application of the results obtained in section 2 we classify the Cohen-Macaulay and unmixed binomial edge ideals $J(G)$ when $G$ is a bicyclic graph, that is the case of deviation 2 .

\section{Preliminaries}

In this section we recall some concepts and notation on graphs and on simplicial complexes that we will use in the article.

Let $G$ be a simple graph with vertex set $V(G)$ and edge set $E(G)$. A subset $C$ of $V(G)$ is called a clique of $G$ if for all $i$ and $j$ belonging to $C$ with $i \neq j$ one has $\{i, j\} \in E(G)$. A vertex of a graph is called a cutpoint if the removal of the vertex increases the number of connected components. A connected subgraph of $G$ that has no cutpoint and is maximal with respect to this property is a block. A block graph $B(G)$ is a graph whose vertices are the blocks of $G$ and two vertices are adjacent whenever the corresponding blocks contain a common cutpoint of $G$. A connected graph is a cactus if its blocks are cycles or edges.

Set $V=\left\{x_{1}, \ldots, x_{n}\right\}$. A simplicial complex $\Delta$ on the vertex set $V$ is a collection of subsets of $V$ such that: $(i)\left\{x_{i}\right\} \in \Delta$ for all $x_{i} \in V$; (ii) $F \in \Delta$ and $G \subseteq F$ imply $G \in \Delta$. An element $F \in \Delta$ is called a face of $\Delta$. A maximal face of $\Delta$ with respect to inclusion is called a facet of $\Delta$. A vertex $i$ of $\Delta$ is called a free vertex of $\Delta$ if $i$ belongs to exactly one facet. The clique complex $\Delta(G)$ of $G$ is the simplicial complex whose faces are the cliques of $G$. Hence a vertex $v$ of a graph $G$ is called free vertex if it belongs to only one clique of $\Delta(G)$.

We need notation and results from [6] (section 3) that we recall for the sake of completeness. Let $T \subseteq[n]$, and let $\bar{T}=[n] \backslash T$. Let $G_{1}, \ldots, G_{c(T)}$ be the connected components of the induced subgraph on $\bar{T}$, namely $G_{\bar{T}}$. For each $G_{i}$, denote by $\widetilde{G}_{i}$ the complete graph on the vertex set $V\left(G_{i}\right)$. We set

$$
P_{T}(G)=\left(\bigcup_{i \in T}\left\{x_{i}, y_{i}\right\}, J\left(\widetilde{G}_{1}\right), \ldots, J\left(\widetilde{G}_{c(T)}\right)\right),
$$

$P_{T}(G)$ is a prime ideal. Then $J(G)$ is a radical ideal and

$$
J(G)=\bigcap_{T \subset[n]} P_{T}(G)
$$

is its primary decomposition (see Corollary 2.2 and Theorem 3.2 of [6]). If there is no possible confusion, we write simply $P_{T}$ instead of $P_{T}(G)$. Moreover, height $P_{T}=n+|T|-c(T)$ (see [6, Lemma 3.1]). We denote by $\mathcal{M}(G)$ the set of minimal prime ideals of $J(G)$.

If each $i \in T$ is a cutpoint of the graph $G_{\bar{T} \cup\{i\}}$, then we say that $T$ is a cutset for $G$. We denote by $\mathcal{C}(G)$ the set of all cutsets for $G$.

Lemma 1.1. 6] $P_{T}(G) \in \mathcal{M}(G)$ if and only if $T \in \mathcal{C}(G)$.

Lemma 1.2. 9] Let $G$ be a connected graph. Then $J(G)$ is unmixed if and only if for all $T \in \mathcal{C}(G)$ we have $c(T)=|T|+1$. 
The following observation gives motivation to consider the block graphs in this context.

Proposition 1.3. Let $J(G)$ be unmixed. Then the block graph $B(G)$ is a tree.

Proof. By Theorem 3.5 of [5] each block of the block graph is a complete graph. We recall that two vertices in $B(G)$ are adjacent when the corresponding blocks contain a common cutpoint of $G$. Moreover for each cutpoint $v,\{v\} \in \mathcal{C}(G)$. By Lemma 1.2 the assertion follows.

We observe the following (see also Remark 3.1.(i) of [2])

Proposition 1.4. Let $v$ be a vertex of $G$ with neighbor set $N(v)=\{u \in$ $V(G) \mid\{u, v\} \in E(G)\}$ and let $G_{\bar{v}}$ the graph such that $V\left(G_{\bar{v}}\right)=V(G)$ and $E\left(G_{\bar{v}}\right)=E(G) \cup\left\{\left\{u_{1}, u_{2}\right\} \mid u_{1} \neq u_{2}, u_{1}, u_{2} \in N(v)\right\}$. Then

$$
\mathcal{C}\left(G_{\bar{v}}\right)=\{T \in \mathcal{C}(G) \mid v \notin T\} .
$$

Proof. If $v$ is a free vertex then $E(G)=E\left(G_{\bar{v}}\right)$. In fact by definition there is only one clique containing $v$, that is all vertices adjacent to $v$ are adjacent to each other. Hence we assume $v$ is not a free vertex in $G$. By definition of the graph $G_{\bar{v}} v$ is a free vertex of $G_{\bar{v}}$. This implies by Proposition 1.1 of [9] that there are no cutsets of $G_{\bar{v}}$ containing $v$.

Let $S \subset V(G)$ such that $v \notin S$, and let

$$
G_{1}, \ldots, G_{c_{1}}
$$

be the connected components of the graph $G_{\bar{S}}$, with $v \in V\left(G_{1}\right)$. Each $\{v, u\} \in E(G)$ either intersects $S$, hence is not in $E\left(G_{\bar{S}}\right)$, or is an edge of $G_{1}$ since it is connected through $v$ in $G_{1}$. In the same way, let $H=G_{\bar{v}}$ and let

$$
H_{1}, \ldots, H_{c_{2}}
$$

be the connected components of the graph $H_{\bar{S}}$, with $v \in V\left(H_{1}\right)$. Each $\{v, u\} \in E(H)$ and each $\left\{u_{1}, u_{2}\right\} \in E(H)$ with $u_{1}, u_{2} \in N(v)$ either intersects $S$ or is an edge of $H_{1}$. This implies that $H_{i}=G_{i}$ for $i=2, \ldots, c_{1}$ and $c_{1}=c_{2}$. Moreover

$$
V\left(G_{i}\right)=V\left(H_{i}\right), \quad \forall i=1, \ldots, c_{1} .
$$

In particular this is true for all the cutsets. That is for all $T \in \mathcal{C}(G)$ then $i \in T$ is a cutpoint of the graph $G_{\bar{T} \cup\{i\}}$, and the number of connected components decreases when $S=T \backslash\{i\}$. The same happens for $H$, hence $T \in \mathcal{C}(H)$. The same argument works in the other direction.

Corollary 1.5. Let $v$ be a vertex of $G$ that is not free vertex and let $G_{\bar{v}}$ the graph such that $V\left(G_{\bar{v}}\right)=V(G)$ and $E\left(G_{\bar{v}}\right)=E(G) \cup\left\{\left\{u_{1}, u_{2}\right\} \mid u_{1} \neq\right.$ $\left.u_{2}, u_{1}, u_{2} \in N(v)\right\}$. Then

$$
J(G)=J\left(G_{\bar{v}}\right) \cap Q_{v}
$$

with $Q_{v}=\bigcap_{T \in \mathcal{C}(G), v \in T} P_{T}(G)$. 
Proof. Let $v \in V(G)$ then $J(G)=Q_{\bar{v}} \cap Q_{v}$ with

$$
Q_{v}=\bigcap_{T \in \mathcal{C}(G), v \in T} P_{T}(G), \quad Q_{\bar{v}}=\bigcap_{T \in \mathcal{C}(G), v \notin T} P_{T}(G) .
$$

Applying Proposition 1.4, we have that the cutsets in $\mathcal{C}\left(G_{\bar{v}}\right)$ are exactly the cutsets in $\mathcal{C}(G)$ not containing $v$. Moreover the connected components induced by any $T$ in $G$ and in $G_{\bar{v}}$ have the same set of vertices as stated in the proof of Proposition 1.4 (see (2) ). Using the notation introduced in the mentioned proof $\widetilde{H}_{i}=\widetilde{G}_{i}$ for each connected component, that is

$$
J\left(G_{\bar{v}}\right)=Q_{\bar{v}} .
$$

Example 1.6. Sometime the ideal $Q_{v}$ in Corollary 1.5 has a natural interpretation. For example let $G=C_{4}$ with vertices $V\left(C_{4}\right)=\{1, \ldots, 4\}$ and edges $\{\{1,2\},\{2,3\},\{3,4\},\{1,4\}\}$. Then we obtain

$$
J(G)=J\left(G_{\overline{1}}\right) \cap Q_{1}=J(D) \cap\left(x_{1}, y_{1}, x_{3}, y_{3}\right)
$$

where $D$, known as diamond graph, has $V(D)=V\left(C_{4}\right)$ and $E(D)=E\left(C_{4}\right) \cup$ $\{\{2,4\}\}$ and the ring $S / Q_{v}$ is related to the isolated vertex 2 and 4 . By similar argument we obtain

$$
J(D)=J\left(D_{\overline{2}}\right) \cap Q_{2}=J\left(K_{4}\right) \cap\left(x_{2}, y_{2}, x_{4}, y_{4}\right)
$$

where $K_{4}$ is the complete graph with 4 vertices. If $G=C_{5}$ such an interpretation fails. Let $V\left(C_{5}\right)=\{1, \ldots, 5\}$ and edges $\{\{1,2\},\{2,3\},\{3,4\},\{4,5\},\{1,5\}\}$. If we consider $J(G)=J\left(G_{\overline{1}}\right) \cap Q_{1}$, then $G_{\overline{1}}$ is the graph obtained adding the edge $\{2,5\}$ to the cycle $C_{5}$, as expected by Corollary 1.5 , but

$$
Q_{1}=\left(x_{1}, y_{1}, f_{2,3}, f_{4,5}, x_{3} x_{4}, x_{3} y_{4}, y_{3} x_{4}, y_{3} y_{4}\right) .
$$

Passing from a block that is not complete to a complete one (see (3), (44) in Example 1.6 and Figure 2), is useful for our aim thanks to the following nice result (see Theorem 1.1 of [4]) that we state using our notation

Theorem 1.7. Let $G$ be a graph whose blocks are complete graphs. The following conditions are equivalent:

(1) $J(G)$ is Cohen-Macaulay;

(2) $J(G)$ is unmixed;

(3) $B(G)$ is a tree.

\section{Classification of Cohen-Macaulay cactus graphs}

In this section we provide a classification of Cohen-Macaulay binomial edge ideal $J(G)$ when $G$ is a cactus graphs. Since a binomial edge ideal $J(G)$ is Cohen-Macaulay (resp. unmixed) if and only if $J(H)$ is Cohen-Macaulay (resp. unmixed) for each connected component $H$ of $G$, we assume from now on that the graph $\mathrm{G}$ is connected unless otherwise stated.

We start by the following 
Proposition 2.1. Let $J(G)$ be an unmixed binomial edge ideal. If a cycle $C_{l}$ is a block of $G$ then $l \in\{3,4\}$.

Proof. We begin observing by [4] and [10] that unmixed binomial edge ideals containing blocks that are cycles of length 3 and 4 exist. We assume that exists a cycle $C_{l}$ in $G$ with $l \geq 5$ and $J(G)$ unmixed. Since $C_{l}$ is a block we represent $G$ as

$$
G=C_{l} \cup\left(\bigcup_{i=1}^{r} G_{i}\right)
$$

where $r \geq 0, G_{i}$ are subgraphs of $G$ for all $i,\left|V\left(G_{i}\right) \cap V\left(C_{l}\right)\right|=1$. Since $C_{l}$ is a block we have that $\{v\}=V\left(G_{i}\right) \cap V\left(C_{l}\right)$ is a cutpoint and all the paths between $u \in V\left(G_{i}\right)$ and $w \in V\left(G_{j}\right)$ with $i \neq j$ pass through $v$ (see Theorem 3.1 of [5]).

Let $V\left(C_{l}\right)=\left\{i_{1}, i_{2}, \ldots, i_{l}\right\}$ such that $\left\{i_{j}, i_{j+1}\right\}$ is an edge of $G$ with $j=1, \ldots, l-1$ and $\left\{i_{1}, i_{l}\right\}$ is an edge of $G$, too. We observe that if $T$ is $\left\{i_{j}, i_{k}\right\}$ such that $\left\{i_{j}, i_{k}\right\} \notin E\left(C_{l}\right)$ then $T \in \mathcal{C}(G)$. Moreover $G_{\bar{T}}$ has at least two connected components induced by the remaining $l-2$ vertices of $C_{l}$. Assuming $G$ is unmixed there are exactly 3 connected components in $G_{\bar{T}}$ and this implies that one of the vertices in $T$ is a cutpoint and the other is not a cutpoint. Thanks to this observation we easily obtain a contradiction. We give the proof for the sake of completeness. We observe that in the cutset $\left\{i_{1}, i_{3}\right\}$ we may assume without loss of generality that $i_{1}$ is the cutpoint and $i_{3}$ is not a cutpoint. By the same argument focusing on $\left\{i_{1}, i_{l-1}\right\}, i_{l-1}$ is not a cutpoint. If we consider $\left\{i_{2}, i_{l-1}\right\}$ and $\left\{i_{3}, i_{l}\right\}$ we obtain that $i_{2}$ and $i_{l}$ are cutpoints. Let $T_{1}=\left\{i_{2}, i_{l}\right\}$ since $G_{\bar{T}_{1}}$ has 4 connected components we obtain a contradiction.

Proposition 2.2. Let $J(G)$ be an unmixed binomial edge ideal. If a cycle $C_{4}$ is a block of $G$ then it satisfies the following $(C 4)$-condition

- there are exactly two cutpoints in $C_{4}$ and they are adjacent.

Proof. By Proposition 2 of [10 we know that a graph satisfying the thesis exists. We prove that all the other cases are not unmixed. Let $V\left(C_{4}\right)=$ $\{1,2,3,4\}$ and let $E\left(C_{4}\right)=\{\{1,2\},\{2,3\},\{3,4\},\{1,4\}\}$. We assume the same representation of $G$ given in (5). Suppose that either $r=0$ or $r=1$ with cutpoint 1 . Then $c(\{2,4\})=2$. Hence it is not unmixed. Let $r \geq 2$ and assume that 1 and 3 are cutpoints then $c(\{1,3\})=4$. Hence $J(G)$ is not unmixed.

Definition 2.3. A graph $G$ is decomposable (resp. indecomposable) if exists (resp. does not exist) a decomposition

$$
G=G_{1} \cup G_{2}
$$

with $\{v\}=V\left(G_{1}\right) \cap V\left(G_{2}\right)$ such that $v$ is a free vertex of $\Delta\left(G_{1}\right)$ and $\Delta\left(G_{2}\right)$. By a recursive decomposition (6) applied to each $G_{1}$ and $G_{2}$, after a finite 


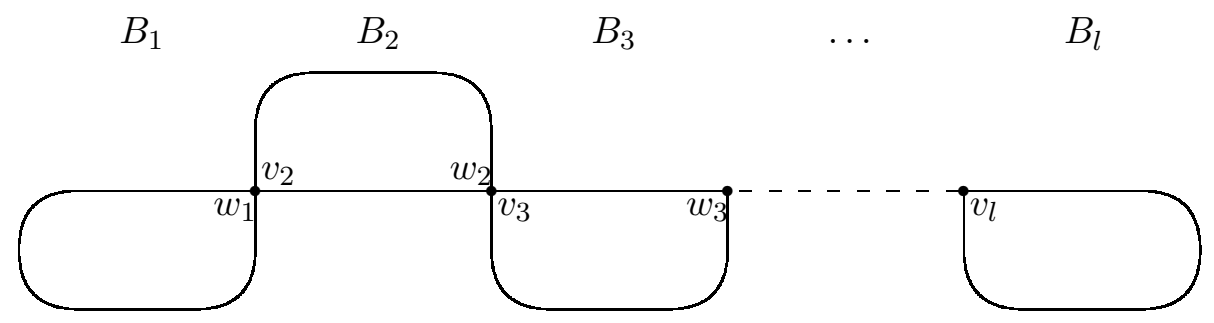

Figure $1 . B(G)$ is a path.

number of steps we obtain

$$
G=G_{1} \cup \cdots \cup G_{r}
$$

where $G_{1}, \ldots, G_{r}$ are indecomposable and for $1 \leq i<j \leq r$ either $V\left(G_{i}\right) \cap$ $V\left(G_{j}\right)=\emptyset$ or $V\left(G_{i}\right) \cap V\left(G_{j}\right)=\{v\}$ and $v$ is a free vertex of $\Delta\left(G_{i}\right)$ and $\Delta\left(G_{j}\right)$. The decomposition (17) is unique up to ordering and we say that $G$ is decomposable into indecomposable graphs $G_{1}, \ldots, G_{r}$.

Lemma 2.4 (See [9]). Let $G$ be a decomposable graph with decomposition $G=G_{1} \cup G_{2}$ and $V\left(G_{1}\right) \cap V\left(G_{2}\right)=\{v\}$. Then $J(G)$ is Cohen-Macaulay (resp. unmixed) if and only if $J\left(G_{1}\right)$ and $J\left(G_{2}\right)$ are Cohen-Macaulay (resp. unmixed).

Our aim is to prove that every Cohen-Macaulay cactus graph $G$ is decomposable into indecomposable graphs $G_{1}, \ldots, G_{r}$ such that the block graph $B\left(G_{i}\right)$ is a path for $1 \leq i \leq r$. Hence it is necessary as a first step to classify the indecomposable Cohen-Macaulay cactus graphs whose block graph is a path. To reach this goal we use from now on the following notation. Let $G$ be a graph such that $B(G)$ is a path defined on the following sets (see Figure 1)

$$
\begin{array}{ll}
\text { vertices of } B(G) & \left\{B_{1}, \ldots, B_{l}\right\} \\
\text { edges of } B(G) & \left\{\left\{B_{i}, B_{i+1}\right\} \mid i=1, \ldots, l-1\right\} \\
\text { cutpoints of } G & \left\{w_{i}=v_{i+1} \mid i=1, \ldots, l-1\right\}
\end{array}
$$

and such that $w_{i}=v_{i+1} \in V\left(B_{i}\right) \cap V\left(B_{i+1}\right)$, with $i=1, \ldots, l-1$.

Lemma 2.5. Let $G$ be a graph such that $B(G)$ is a path. We use notation (8). If $\left|V\left(B_{i}\right)\right| \geq 3$ for all $1<i<l$ then the power set of $\left\{w_{1}, \ldots, w_{l-1}\right\}$ is a subset of $\mathcal{C}(G)$.

Proof. We use induction on $t$ the cardinality of $T \in \mathcal{C}(G)$. If $t=1$ since each $w_{i}$ is a cutpoint of $G$ the claim follows. Let $2 \leq t<l-1$ and

$$
T=\left\{w_{i_{1}}, w_{i_{2}}, \ldots w_{i_{t}}\right\} \text { with } 1 \leq i_{1}<i_{2}<\cdots<i_{t} \leq l-1 .
$$

Let $w_{j} \in\left\{w_{1}, \ldots, w_{l-1}\right\} \backslash T$ and assume without loss of generality that $j<i_{1}$ and $i_{1} \geq 2$. We observe that the graph $G_{\bar{T}}$ has the connected component $H=B_{1} \cup \ldots \cup B_{i_{1}-1} \cup B_{i_{1}}^{\prime}$ where $B_{i_{1}}^{\prime}$ is obtained removing the vertex $w_{i_{1}}$ from the block $B_{i_{1}}$. Since by hypothesis $B_{i_{1}}$ has more than two vertices, $B_{i_{1}}^{\prime}$ 
contains at least one vertex, $v_{i_{1}}^{\prime}$, that is not a cutpoint in $G$. Hence $H_{\overline{\left\{w_{j}\right\}}}$ has two connected components: one containing a vertex in $V\left(B_{1}\right) \backslash\left\{w_{1}\right\}$ and one containing the vertex $v_{i_{1}}^{\prime}$. That is $w_{j}$ is a cutpoint of $G_{\bar{T}}$ and $T \cup\left\{w_{j}\right\} \in \mathcal{C}(G)$.

By Proposition 2.1, Example 1.6 and Theorem 1.7 is useful for our aim to compute the primary decompositions of $J(G)$ whose blocks are complete graphs, cycles $C_{4}$ and diamond graphs $D$ (see also Figure 2).

Proposition 2.6. Let $G$ be a graph such that $B(G)$ is a path. We use notation (8). Let $B_{1}=K_{m_{1}}, B_{l}=K_{m_{l}}$ with $m_{1}, m_{l} \geq 2$ and

$$
B_{i} \in\left\{C_{4}, D, K_{m_{i}} \mid m_{i} \geq 3\right\} \text { for } 2 \leq i \leq l-1
$$

with the following labelling on the vertices of the blocks $B_{i} \in\left\{C_{4}, D\right\}$ :

- $V\left(C_{4}\right)=\left\{v_{i}, v_{i}^{\prime}, w_{i}, w_{i}^{\prime}\right\}, E\left(C_{4}\right)=\left\{\left\{v_{i}, w_{i}\right\},\left\{v_{i}, v_{i}^{\prime}\right\},\left\{v_{i}^{\prime}, w_{i}^{\prime}\right\},\left\{w_{i}, w_{i}^{\prime}\right\}\right\}$;

- $V(D)=\left\{v_{i}, v_{i}^{\prime}, w_{i}, f_{i}\right\}, E(D)=\left\{\left\{v_{i}, w_{i}\right\},\left\{v_{i}, v_{i}^{\prime}\right\},\left\{v_{i}^{\prime}, f_{i}\right\},\left\{w_{i}, f_{i}\right\},\left\{v_{i}^{\prime}, w_{i}\right\}\right\}$.

Then $T \in \mathcal{C}(G)$ if and only if $T \subseteq V \sqcup V^{\prime}$ where $V=\left\{w_{1}, \ldots, w_{l-1}\right\}$,

$$
V^{\prime}=\left(\bigcup_{B_{i}=C_{4}}\left\{v_{i}^{\prime}, w_{i}^{\prime}\right\}\right) \cup\left(\bigcup_{B_{i}=D}\left\{v_{i}^{\prime}\right\}\right)
$$

and satisfying the following conditions:

(1) if $v_{i}^{\prime} \in T$ then $w_{i} \in T$ and $v_{i} \notin T$;

(2) if $w_{i}^{\prime} \in T$ then $v_{i} \in T$ and $w_{i} \notin T$.

Proof. For the sake of completeness we give the equivalent conditions to (1) and (2) that are:

(1') if $v_{i} \in T$ or $w_{i} \notin T$ then $v_{i}^{\prime} \notin T$;

(2') if $w_{i} \in T$ or $v_{i} \notin T$ then $w_{i}^{\prime} \notin T$.

Suppose $T$ is a cutset. Then $v \in T$ is not a free vertex. Hence it follows $T \subseteq$ $V \sqcup V^{\prime}$. In fact a vertex $f_{i}$ in a diamond graph is a free vertex in the clique $\left\{v_{i}^{\prime}, w_{i}, f_{i}\right\}$ and the same holds for all the vertices that are not cutpoints in a block that is a complete graph. Moreover, if $T \subseteq V$ then it satisfies condition 1. and 2. in a trivial way. Hence we assume $v^{\prime} \in T$ with $v^{\prime} \in V^{\prime}$. That is either $v^{\prime}=v_{i}^{\prime} \in V\left(B_{i}\right)$ with $B_{i} \in\left\{C_{4}, D\right\}$ or $v^{\prime}=w_{i}^{\prime} \in V\left(B_{i}\right)$ with $B_{i}=C_{4}$. We consider only the case $v^{\prime}=v_{i}^{\prime} \in V\left(B_{i}\right)$ with $B_{i}=C_{4}$ since the other cases follow by similar argument. Since $T$ is a cutset, the graph $G_{S}$ with $S=\bar{T} \cup\left\{v_{i}^{\prime}\right\}$ has a cutpoint in $v_{i}^{\prime}$. Hence the two vertices $v_{i}$ and $w_{i}^{\prime}$ that are adjacent to $v_{i}^{\prime}$ in $G$ must belong to $G_{S}$, that is $v_{i}$ and $w_{i}^{\prime}$ are not in $T$. By the same reason since $v_{i}^{\prime}$ is a cutpoint in $G_{S}$ and since $w_{i}$ is adjacent to $v_{i}$ and $w_{i}^{\prime}$ in $G$, too, then $w_{i} \notin S$ and $w_{i} \in T$. That is condition (1) is satisfied.

Now suppose $T \subseteq V \sqcup V^{\prime}$ satisfying conditions (1) and (2) and let $n^{\prime}=\left|V^{\prime}\right|$. We prove that $T$ is a cutset by induction on $n^{\prime}$. If $n^{\prime}=0$ that is $T \subseteq V$, then it is a cutset by Lemma 2.5 . 
Let $n^{\prime} \geq 1$ and let $v_{2}^{\prime} \in V^{\prime}$ then $B_{2} \in\left\{C_{4}, D\right\}$ (see Figure 2). We assume $B_{2}=C_{4}$ since the other case is similar. By conditions (1) and (2'). $w_{2} \in T$ and neither $v_{2}$ nor $w_{2}^{\prime}$ belongs to $T$. That is the graph $G_{\bar{T}}$ contains 2 connected components, $B_{1}$ and the isolated vertex $w_{2}^{\prime}$, and a subgraph of $H=B_{3}^{\prime} \cup B_{4} \cup \cdots \cup B_{l}$, where $B_{3}^{\prime}$ is obtained removing the vertex $w_{2}=v_{3}$ from $B_{3}$.

We claim that $T^{\prime}=T \backslash\left\{v_{2}^{\prime}, w_{2}\right\}$ is a cutset of $H$. Moreover $H_{\overline{T^{\prime}}}$ has a connected component containing a vertex in $B_{3}$ that is adjacent to $w_{2}$. By the claim it easily follows that $T$ is a cutset of $G$ and $G_{\bar{T}}$ contains the following connected components: $B_{1}$, the isolated vertex $w_{2}^{\prime}$, and the connected components of $H_{\overline{T^{\prime}}}$.

If $B_{3}$ is a complete graph then $B_{3}^{\prime}$ is a complete graph, too. Hence $H$ satisfies the hypothesis of our Proposition and $T^{\prime}$ is a cutset by induction hypothesis. If $B_{3}=D$ then $B_{3}^{\prime}$ is the complete graph $K_{3}$. Moreover $v_{3}^{\prime} \notin T$ by condition (1') applied to $w_{2}=v_{3} \in T$. Hence the set $T^{\prime}$ contains at most the cutpoint $w_{3}$. Also in this case the graph $H$ satisfies the hypothesis of our Proposition and $T^{\prime}$ is a cutset by induction hypothesis. If $B_{3}=C_{4}$ then $B_{3}^{\prime}$ is a path of length 2 whose edges are

$$
\left\{v_{3}^{\prime}, w_{3}^{\prime}\right\},\left\{w_{3}^{\prime}, w_{3}\right\} .
$$

We note that also in this case $v_{3}^{\prime} \notin T$ by condition (1'), and $T$ contains at most one of the vertices $w_{3}$ and $w_{3}^{\prime}$. In fact $v_{3}^{\prime}$ is the free vertex of the path and only one of the cutpoints $w_{3}, w_{3}^{\prime}$ can appear in $T^{\prime}$. Moreover we can apply induction hypothesis on the subgraph $H^{\prime}$ obtained removing $v_{3}^{\prime}$ from $H$.

By similar argument to the previous case we obtain the assertion under the assumption $w_{2}^{\prime} \in V^{\prime}$. In this case $B_{2}=C_{4}, v_{2} \in T$ and $G_{\bar{T}}$ contains the following connected components: the isolated vertex $v_{2}^{\prime}, B_{1}^{\prime}$, obtained removing the vertex $v_{2}=w_{1}$ from $B_{1}$, and the connected components of $H_{\overline{T^{\prime}}}$ with $H=B_{3} \cup \cdots \cup B_{l}$ and $T^{\prime}=T \backslash\left\{w_{2}^{\prime}, v_{2}\right\}$.

Corollary 2.7. Let $G$ be a graph that satisfies the hypothesis of Proposition 2.6. Then $J(G)$ is unmixed.

Proof. By Lemma 1.2 it is sufficient to show that $c(T)=|T|+1$ for all $T \in \mathcal{C}(G)$. Thanks to Lemma 2.5, $T=\left\{w_{i_{1}}, w_{i_{2}}, \ldots w_{i_{t}}\right\} \subseteq\left\{w_{1}, \ldots, w_{l-1}\right\}$ with $1 \leq i_{1}<i_{2}<\cdots<i_{t} \leq l-1$ is a cutset of $G$. We note that

$$
G_{\bar{T}}=G_{1} \sqcup G_{2} \sqcup \cdots \sqcup G_{t+1}
$$

where $G_{1}=B_{1} \cup \cdots \cup B_{i_{1}-1} \cup B_{i_{1}}^{\prime \prime}, G_{t+1}=B_{i_{t}+1}^{\prime} \cup B_{i_{t}+2} \cup \cdots \cup B_{l}$ and

$$
G_{j}=B_{i_{j}+1}^{\prime} \cup B_{i_{j}+2} \cup \cdots \cup B_{i_{j+1}-1} \cup B_{i_{j+1}}^{\prime \prime} \text { with } j=2, \ldots, t
$$

and $B_{i}^{\prime \prime}$ is the block obtained removing $w_{i}$ from $B_{i}, B_{i}^{\prime}$ is the block obtained removing $w_{i-1}=v_{i}$ from $B_{i}$. We easily observe that $G_{i}$ is connected for $1 \leq i \leq t+1$. Hence $c(T)=t+1$. Now let $T \sqcup T^{\prime} \in \mathcal{C}(G)$ with $T=$ $\left\{w_{i_{1}}, w_{i_{2}}, \ldots w_{i_{t}}\right\} \subseteq\left\{w_{1}, \ldots, w_{l-1}\right\}$ and $T^{\prime} \cap\left\{w_{1}, \ldots, w_{l-1}\right\}=\emptyset$ with $t^{\prime}=$ 


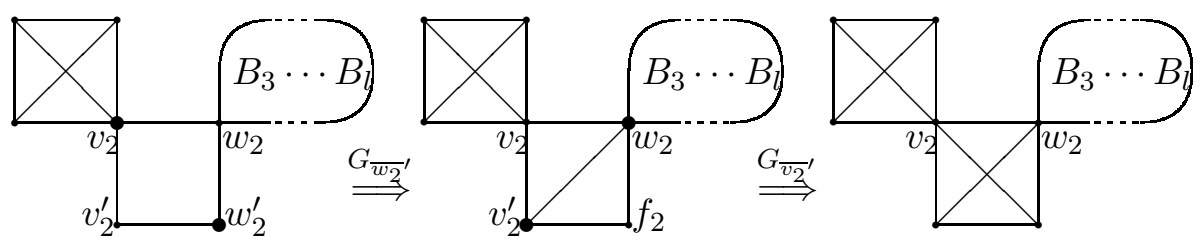

Figure 2. Cases $B_{2} \in\left\{C_{4}, D, K_{4}\right\}$ of Theorem 2.8

$\left|T^{\prime}\right|>0$. This implies that $v^{\prime} \in T^{\prime}$ is either $v_{i}^{\prime}$ or $w_{i}^{\prime}$ of $B_{i} \in\left\{C_{4}, D\right\}$. If we focus on $T$ the representation of $G_{\bar{T}}$ in (9) holds in this case, too. Without loss of generality let $v^{\prime} \in V\left(G_{1}\right)$. Since the only cutpoint that induces the connected component $G_{1}$ is $w_{i_{1}}$, by condition (1) of Proposition 2.6, $v^{\prime}=v_{i_{1}}^{\prime}$. Hence

$$
G_{\overline{T \cup\left\{v_{i_{1}}^{\prime}\right\}}}=\left(G_{1}\right)_{\overline{\left\{v_{i_{1}}^{\prime}\right\}}} \sqcup G_{2} \sqcup \cdots \sqcup G_{t+1}
$$

where $\left(G_{1}\right)_{\left.\overline{\left\{i_{i_{1}}^{\prime}\right.}\right\}}=B_{1} \cup \cdots \cup B_{i_{1}-1} \sqcup\{v\}$ where $v=f_{i_{1}}$ if $B_{i_{1}}=D$ or $v=w_{i_{1}}^{\prime}$ if $B_{i_{1}}=C_{4}$ and $c\left(T \cup\left\{v_{i_{1}}^{\prime}\right\}\right)=(t+1)+1$ where the last summand is induced by the isolated vertex $v$. The same argument holds for all $v^{\prime} \in T^{\prime}$.

Theorem 2.8. Let $G$ be a graph that satisfies the hypothesis of Proposition 2.6. Then $J(G)$ is Cohen-Macaulay.

Proof. We start observing that $\operatorname{dim} S / J(G)=n+1$. This follows by Corollary 2.7 and the formula (see [6])

$$
\operatorname{dim} S / J(G)=\max \{n-|T|+c(T)\} .
$$

Hence it is sufficient to prove that $\operatorname{depth} S / J(G) \geq n+1$ using induction on $l$, the number of blocks of $G$. Our strategy is to focus on the block $B_{2}$. In particular when $B_{2} \in\left\{D, C_{4}\right\}$ (see Figure 2) we consider the vertex $v^{\prime} \in\left\{v_{2}^{\prime}, w_{2}^{\prime}\right\}$ and the following exact sequence

$$
0 \longrightarrow S / J(G) \longrightarrow S / Q_{v^{\prime}} \oplus S / J\left(G^{\prime}\right) \longrightarrow S /\left(Q_{v^{\prime}}+J\left(G^{\prime}\right)\right) \longrightarrow 0
$$

with $G^{\prime}=G_{\overline{v^{\prime}}}$. By Corollary 2.5, $J\left(G^{\prime}\right)$ is a binomial edge ideal and the graph $G^{\prime}$ satisfies the hypothesis of our Theorem but with a second block that has less cutsets than $B_{2}$ (see Figure (2) ). Moreover $S / Q_{v^{\prime}}$ (respectively $S / Q_{v^{\prime}}+J\left(G^{\prime}\right)$ ) is a tensor product of 3 (respectively 2) quotient rings whose definining ideals are binomial edge ideals, and it is Cohen-Macaulay using induction. To obtain this goal we have three cases to study: $B_{2}=K_{m_{2}}$, $B_{2}=D$ and $B_{2}=C_{4}$. We start induction with $l=3$.

Case $B_{2}=K_{m_{2}}$. $S / J(G)$ is Cohen-Macaulay by Theorem 1.1 of [4].

Case $B_{2}=D$. We set $G^{\prime}=G_{{\overline{v_{2}}}^{\prime}}$. By Proposition 1.4 we have $E\left(G^{\prime}\right)=$ $E(G) \cup\left\{\left\{v_{2}, f_{2}\right\}\right\}$, that is the second block in $G^{\prime}$ is the complete graph on the vertices $\left\{v_{2}, v_{2}^{\prime}, w_{2}, f_{2}\right\}$. By Corollary [1.5, $J(G)=J\left(G^{\prime}\right) \cap Q_{v_{2}^{\prime}}$, where $Q_{v_{2}^{\prime}}=P_{\left\{v_{2}^{\prime}, w_{2}\right\}}=\left(x_{v_{2}^{\prime}}, y_{v_{2}^{\prime}}\right)+\left(x_{w_{2}}, y_{w_{2}}\right)+J\left(B_{1}\right)+J\left(B_{3}^{\prime}\right)$ and $B_{3}^{\prime}$ is obtained by removing the vertex $w_{2}=v_{3}$ from the complete graph $B_{3}$. By Depth 
Lemma applied on the sequence (12) with $v^{\prime}=v_{2}^{\prime}$ we obtain the assertion. In fact $G^{\prime}$ is Cohen-Macaulay by the case $B_{2}=K_{m_{2}}$ and its depth is $n+1$. Let $S^{\prime}=S /\left(x_{v_{2}^{\prime}}, y_{v_{2}^{\prime}}, x_{w_{2}}, y_{w_{2}}\right)$, we obtain

$$
\begin{aligned}
& S / Q_{v_{2}^{\prime}} \cong S^{\prime} / J\left(B_{1}\right)+J\left(B_{3}^{\prime}\right) \cong \\
& \quad \cong \frac{K\left[\left\{x_{i}, y_{i}\right\}: i \in V\left(B_{1}\right)\right]}{J\left(B_{1}\right)} \otimes K\left[x_{f_{2}}, y_{f_{2}}\right] \otimes \frac{K\left[\left\{x_{i}, y_{i}\right\}: i \in V\left(B_{3}^{\prime}\right)\right]}{J\left(B_{3}^{\prime}\right)}
\end{aligned}
$$

where the 3 quotient rings associated to the complete graphs $B_{1}, B_{3}^{\prime}$ and the isolated vertex $f_{2}$ are Cohen-Macaulay. Using the formula (11) for each ring, and adding the results thanks to (13), we obtain

$$
\operatorname{depth} S / Q_{v_{2}^{\prime}}=\mid\left(V\left(B_{1}\right)+1\right)+(1+1)+\left(\left|V\left(B_{3}^{\prime}\right)\right|+1\right)=n+1 .
$$

By the same argument depth $S /\left(Q_{v_{2}^{\prime}}+J\left(G^{\prime}\right)\right)=n$. In fact

$$
\begin{aligned}
S / Q_{v_{2}^{\prime}}+ & J\left(G^{\prime}\right) \cong S^{\prime} / J\left(B_{1} \cup E^{\prime}\right)+J\left(B_{3}^{\prime}\right) \cong \\
& \cong \frac{K\left[\left\{x_{i}, y_{i}\right\}: i \in V\left(B_{1}\right) \cup\left\{f_{2}\right\}\right]}{J\left(B_{1} \cup E\right)} \otimes \frac{K\left[\left\{x_{i}, y_{i}\right\}: i \in V\left(B_{3}^{\prime}\right)\right]}{J\left(B_{3}^{\prime}\right)}
\end{aligned}
$$

where $E$ is the edge $\left\{w_{1}=v_{2}, f_{2}\right\}$. Hence $J\left(B_{1} \cup E\right)$ is Cohen-Macaulay by Theorem 1.1 of [4]. By Depth Lemma the assertion follows.

Case $B_{2}=C_{4}$. We set $v^{\prime}=w_{2}^{\prime}$ and $G^{\prime}=G_{\overline{\bar{w}_{2}}}$. By Proposition 1.4 we have $E\left(G^{\prime}\right)=E(G) \cup\left\{\left\{v_{2}^{\prime}, w_{2}\right\}\right\}$, that is the second block in $G^{\prime}$ is a diamond, hence $J\left(G^{\prime}\right)$ is Cohen-Macaualy by the case $B_{2}=D$. Moreover $\operatorname{depth} S / Q_{v^{\prime}}=n+1$ and depth $S / Q_{v^{\prime}}+J\left(G^{\prime}\right)=n$. In fact $Q_{v^{\prime}}=P_{\left\{v_{2}, w_{2}^{\prime}\right\}}=$ $\left(x_{w_{2}^{\prime}}, y_{w_{2}^{\prime}}\right)+\left(x_{v_{2}}, y_{v_{2}}\right)+J\left(B_{1}^{\prime}\right)+J\left(B_{3}\right)$, where $B_{1}^{\prime}$ is the complete graph obtained removing the vertex $v_{2}=w_{1}$ from the graph $B_{1}$. Using a representation of $S / Q_{v^{\prime}}$ similar to (13) we easily obtain the assertion. Moreover $Q_{v^{\prime}}+J\left(G^{\prime}\right)=\left(x_{w_{2}^{\prime}}, y_{w_{2}^{\prime}}\right)+\left(x_{v_{2}}, y_{v_{2}}\right)+J\left(B_{1}^{\prime}\right)+J\left(B_{3} \cup E\right)$ where $E$ is the edge $\left\{v_{2}^{\prime}, w_{2}\right\}$. Also in this case we obtain the assertion using a representation of $S / Q_{v^{\prime}}+J\left(G^{\prime}\right)$ equivalent to the one used in (14).

Let $l>3$. Case $B_{2}=K_{m_{2}}$. By Lemma 2.4 applied on the graphs $G_{1}=B_{1}$ and $G_{2}=B_{2} \cup \cdots \cup B_{l}$, since $G_{1}$ is a complete graph and $G_{2}$ is Cohen-Macaulay by induction hypothesis, we obtain that $G$ is CohenMacaulay.

Case $B_{2}=D$. As in the case $B_{2}=D$ with $l=3$, setting $G^{\prime}=G_{\overline{v_{2}}}$, the second block in $G^{\prime}$, namely $B_{2}^{\prime}$, is the complete graph on the vertices $\left\{v_{2}, v_{2}^{\prime}, w_{2}, w_{2}^{\prime}\right\}$, hence

$$
G^{\prime}=B_{1} \cup B_{2}^{\prime} \cup B_{3} \cup \cdots \cup B_{l} .
$$

Therefore $J\left(G^{\prime}\right)$ is Cohen-Macaulay using induction hypothesis and Lemma 2.4 applied on $B_{1}$ and $B_{2}^{\prime} \cup B_{3} \cup \cdots \cup B_{l}$. By Corollary 1.5 $Q_{v_{2}^{\prime}}=\bigcap_{T \in \mathcal{C}(G), v_{2}^{\prime} \in T} P_{T}(G)$. Thanks to condition (1) of Proposition 2.6 for each $T \in \mathcal{C}(G)$ with $v_{2}^{\prime} \in T$ then $w_{2} \in T$ and $v_{2} \notin T$. Hence $Q_{v_{2}^{\prime}}=J\left(B_{1}\right)+\left(x_{v_{2}^{\prime}}, y_{v_{2}^{\prime}}, x_{w_{2}}, y_{w_{2}}\right)+J$. We claim that $J=J(H)$, where $H=B_{3}^{\prime} \cup B_{4} \cup \cdots \cup B_{l}$, with $B_{3}^{\prime}$ obtained removing the vertex $w_{2}=v_{3}$ from $B_{3}$. By the claim, and defining 
$S^{\prime}=S /\left(x_{v_{2}^{\prime}}, y_{v_{2}^{\prime}}, x_{w_{2}}, y_{w_{2}}\right)$, we obtain a representation of $S / Q_{v_{2}^{\prime}}$ by tensor product similar to (13), that is

$$
\frac{K\left[\left\{x_{i}, y_{i}\right\}: i \in V\left(B_{1}\right)\right]}{J\left(B_{1}\right)} \otimes K\left[x_{f_{2}}, y_{f_{2}}\right] \otimes \frac{K\left[\left\{x_{i}, y_{i}\right\}: i \in V(H)\right]}{J(H)},
$$

and using induction hypothesis on $H$ we obtain the assertion. The claim follows proving the condition

$$
T \in \mathcal{C}(H) \text { if and only if } T \cup\left\{v_{2}^{\prime}, w_{2}\right\} \in \mathcal{C}(G) .
$$

Proof of (15). Case $B_{3}=D$. Let $B_{3}^{\prime}=K_{3}$ on the vertices $\left\{v_{3}^{\prime}, f_{3}, w_{3}\right\}$, then $H$ satisfies Proposition 2.6. Let $T \in \mathcal{C}(H)$. To prove that $T \cup\left\{v_{2}^{\prime}, w_{2}\right\} \in \mathcal{C}(G)$ it is enough to check conditions (1) and (2) for all the vertices $v^{\prime} \in T^{\prime}=$ $\left(T \cup\left\{v_{2}^{\prime}, w_{2}\right\}\right) \backslash\left\{w_{1}, \ldots, w_{l-1}\right\}$. If $v^{\prime} \in V\left(B_{2}\right) \cap T^{\prime}$ then $v^{\prime}=v_{2}^{\prime}$ and it satisfies condition (1). If $v^{\prime} \in V\left(B_{i}\right) \cap T^{\prime}$ with $4 \leq i \leq l$ then it satisfies condition 1 . and 2. with respect to $G$ since it satisfies the same conditions with respect to $H$. We end this implication observing that $V\left(B_{3}\right) \cap T^{\prime}=\emptyset$. If $T \subseteq V(H)$ with $T \notin \mathcal{C}(H)$ either there exists a block in $\left\{B_{4}, \ldots, B_{l}\right\}$ such that either (1) or $(2)$ is not satisfied or there exists $v \in V\left(B_{3}^{\prime}\right)$ with $v \neq w_{3}$. In both cases $T \cup\left\{v_{2}^{\prime}, w_{2}\right\}$ does not satisfy (1) and (2) with respect to $G$.

The cases $B_{3} \in\left\{K_{m_{3}}, C_{4}\right\}$ follow by similar argument. We only point out some facts when $B_{3}=C_{4} . \quad B_{3}^{\prime}$ is a path defined on the vertex set $\left\{v_{3}^{\prime}, w_{3}^{\prime}, w_{3}\right\}$ and edges $\left\{v_{3}^{\prime}, w_{3}^{\prime}\right\},\left\{w_{3}^{\prime}, w_{3}\right\}$. In this case is useful to consider $H=E \cup H^{\prime}$ where $E$ is the edge $\left\{v_{3}^{\prime}, w_{3}^{\prime}\right\}$ and $H^{\prime}$ is obtained removing the vertex $v_{3}^{\prime}$ from $H$. Then $H^{\prime}$ satisfies the hypothesis of the Proposition and it is Cohen-Macaulay by induction. By Lemma 2.4 applied to $H^{\prime}$ and $E$ we obtain that $J(H)$ is Cohen-Macaulay, too. Moreover by Lemma 2.3 of [9] we have

$$
\mathcal{C}(H)=\mathcal{C}\left(H^{\prime}\right) \cup\left\{T \cup\left\{w_{3}^{\prime}\right\}: T \in \mathcal{C}\left(H^{\prime}\right) \text { with } w_{3} \notin T\right\} .
$$

Observe that the graph studied in this case is decomposable with respect to the vertex $v_{2}$. Hence also the graphs

$$
B_{2} \cup \cdots \cup B_{l} \text { with } B_{2}=D \text { is Cohen-Macaulay. }
$$

Case $B_{2}=C_{4}$. Let $G^{\prime}=G_{{\overline{w_{2}}}^{\prime}}$. As in the case $B_{2}=C_{4}$ and $l=3$, the second block of $G^{\prime}$ is $D$. Then $J\left(G^{\prime}\right)$ is Cohen-Macaulay by the case $B_{2}=D$ with $l>3$, and $J(G)=J\left(G^{\prime}\right) \cap Q_{w_{2}^{\prime}}$ with $Q_{w_{2}^{\prime}}=\bigcap_{T \in \mathcal{C}(G), w_{2}^{\prime} \in T} P_{T}(G)$. We claim that $Q_{w_{2}^{\prime}}=J\left(B_{1}^{\prime}\right)+\left(x_{w_{2}^{\prime}}, y_{w_{2}^{\prime}}\right)+\left(x_{v_{2}}, y_{v_{2}}\right)+J(H)$ where $B_{1}^{\prime}$ is the complete graph obtained removing $v_{2}=w_{1}$ from $B_{1}$. Moreover, using the notation introduced in Proposition 1.4,

$$
H=\left(B_{3} \cup B_{4} \cup \cdots \cup B_{l}\right)_{\overline{v_{3}}}=\left(B_{3}\right)_{\overline{v_{3}}} \cup B_{4} \cup \cdots \cup B_{l} .
$$

By the claim it follows, defining $S^{\prime}=S /\left(x_{v_{2}}, y_{v_{2}}, x_{w_{2}^{\prime}}, y_{w_{2}^{\prime}}\right)$, the following representation of $S / Q_{w_{2}^{\prime}}$

$$
\frac{K\left[\left\{x_{i}, y_{i}\right\}: i \in V\left(B_{1}^{\prime}\right)\right]}{J\left(B_{1}^{\prime}\right)} \otimes K\left[x_{v_{2}^{\prime}}, y_{v_{2}^{\prime}}\right] \otimes \frac{K\left[\left\{x_{i}, y_{i}\right\}: i \in V(H)\right]}{J(H)} .
$$




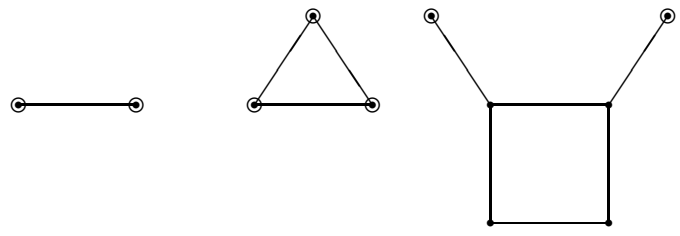

Figure 3. Tree and unicyclic Cohen-Macaulay indecomposable graphs.

We focus on the last factor since the other ones are exactly equivalent to the ones already studied. The block $\left(B_{3}\right)_{\overline{v_{3}}}$ is either a complete graph or a diamond graph with $v_{3}$ a free vertex. In both cases it is Cohen-Macaulay by induction hypothesis and (16). The claim follows by the condition

$$
T \in \mathcal{C}(H) \text { if and only if } T \cup\left\{v_{2}, w_{2}^{\prime}\right\} \in \mathcal{C}(G) .
$$

To prove (17) we use Proposition 2.6 and similar arguments to the ones used to prove (15).

Lemma 2.9. Let $G$ be an indecomposable cactus graph such that $B(G)$ is a path. We use notation (8). The following conditions are equivalent:

(1) $J(G)$ is Cohen-Macaulay;

(2) $J(G)$ is unmixed;

(3) One of the following 2 cases occurs

(a) $G \in\left\{K_{2}, C_{3}\right\}$.

(b) $G$ has $C_{4}$ subgraphs that satisfy the (C4)-condition, $l \geq 3$ and

(i) $B_{1}, B_{l} \in\left\{C_{3}, K_{2}\right\}$,

(ii) $B_{2}$ and $B_{l-1}$ are $C_{4}$,

(iii) $B_{i} \in\left\{C_{3}, C_{4}\right\}$ for $3 \leq i \leq l-2$ and if $B_{i}=C_{3}$ then $B_{i+1}=C_{4}$.

Proof. (1) $\Rightarrow(2)$. It is a known fact. (2) $\Rightarrow(3)$. By Proposition 2.1 only $K_{2}$, $C_{3}$ and $C_{4}$ are admissible blocks. Since every vertex of a complete graph is a free vertex, we observe that every cactus graph whose blocks are $K_{2}$ and $C_{3}$ and not $C_{4}$ is Cohen-Macaulay and is decomposable in single blocks. Now suppose that the block graph is a path containing one or more blocks that are $C_{4}$. By Proposition 2.2 a block $C_{4}$ has two cutpoints, thus neither $B_{1}$ nor $B_{l}$ is $C_{4}$. Since by hypothesis $G$ is indecomposable, two complete graphs cannot be adjacent in $B(G)$. We end observing that if a $K_{2}$ is between two cycles $C_{4}$ it is not unmixed (see also Remark 4.7 of [2]). (3) $\Rightarrow(1)$. The implication follows applying Theorem 2.8 .

Example 2.10. Thanks to Lemma 2.9 we obtain the Cohen-Macaulay indecomposable graph of trees and unicyclic graphs shown in Figure 3. The bicyclic with the same properties are in Figure 4. From now on we underline the free vertices by a circle around them. 

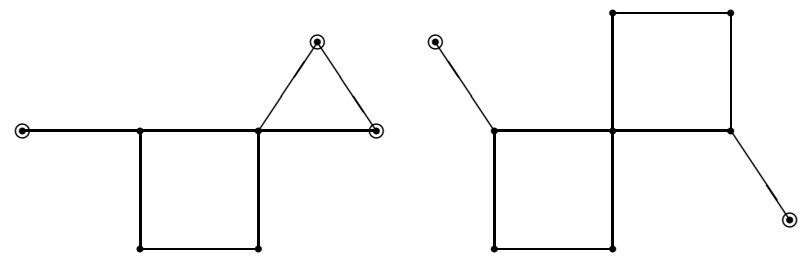

Figure 4. Bicyclic Cohen-Macaulay indecomposable cactus graphs.

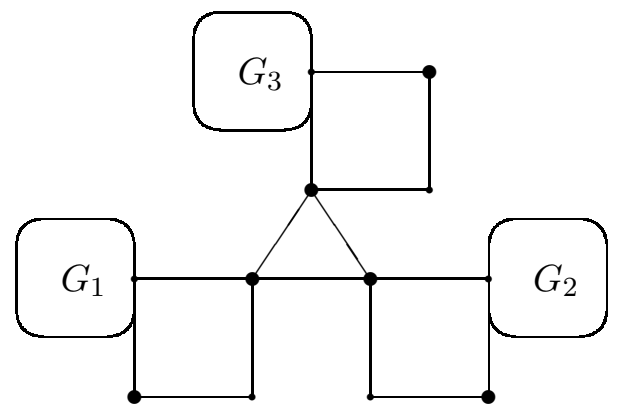

Figure 5. A non unmixed cactus graphs with $B(G)$ that is a tree.

Theorem 2.11. Let $G$ be a cactus graph. We use notation (8). The following conditions are equivalent

(1) $J(G)$ is Cohen-Macaulay;

(2) $J(G)$ is unmixed;

(3) $B(G)$ is a tree, $G$ is decomposable into indecomposable graphs $G_{1}, \ldots, G_{r}$, and such that $B\left(G_{i}\right)$ is a path and $G_{i}$ satisfies one of the equivalent conditions of Lemma 2.9 for $1 \leq i \leq r$.

Proof. (1) $\Rightarrow(2)$. It is a known fact. $(3) \Rightarrow(1)$. It follows by Lemma 2.4. $(2) \Rightarrow(3)$. By Proposition 1.3 the block graph of $G$ is a tree. Let $t$ be the number of vertices of $B(G)$ having degree greater than two. We make induction on $t$. If $t=0$ then the block graph $B(G)$ is a path and the assertion follows by Lemma 2.4 and Lemma 2.9. Let $t>0$ and let $B$ be a a vertex of $B(G)$ whose degree is greater than 2. By Proposition 2.1 and Proposition 2.2, $B=C_{3}$. Suppose that $C_{3}$ has a vertex $v$ such that $\{v\}=V\left(G_{1}\right) \cap V\left(G_{2}\right), G=G_{1} \cup G_{2}$ and $v$ is a free vertex of $\Delta\left(G_{1}\right)$ and $\Delta\left(G_{2}\right)$. Since $G_{1}$ and $G_{2}$ are cactus with number of blocks of degree greater than 2 less than $t$, by induction hypothesis we are done. Suppose by contradiction that in each of the 3 vertices of $C_{3}$ the graph $G$ is not decomposable. Then $C_{3}$ is adjacent to 3 blocks $C_{4}$ as in figure 5 , where $G_{1}$, $G_{2}$ and $G_{3}$ are intended as cactus subgraphs of $G$. We observe that the set $T$ containing the 6 vertices, indicated as filled dots in figure, is a cutset. But $G_{\bar{T}}$ has 6 components. Hence $J(G)$ is not unmixed. Contradiction. 


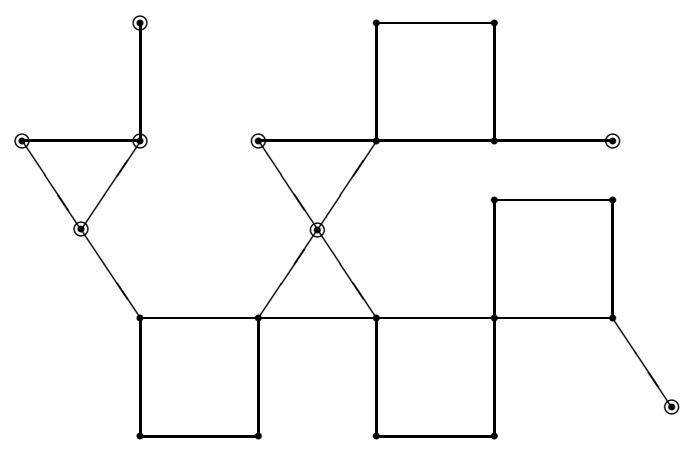

Figure 6. A Cohen-Macaulay cactus graphs.

Example 2.12. In figure [6] we have that $G$ is the union of 4 indecomposable Cohen-Macaulay cactus graphs joined by free vertices (surrounded by a circle). That are : a $K_{2} ; a C_{3}$; one containing a $K_{2}, a C_{3}$ and a $C_{4}$; one containing $2 K_{2}$, one $C_{3}$ and $3 C_{4}$.

\section{Classification of Cohen-Macaulay bicyclic graphs}

In this section thanks to the classification of Cohen-Macaulay binomial edge ideals of cactus graphs we classify the ones that are bicyclic, namely the ideals of deviation 2. In fact a cactus graph having 2 cycles as blocks is in particular a bicyclic graph. Hence we focus our attention on bicyclic graphs $G$ that are not cactus. In this case there exists one block $B_{1}$ in $G$ such that

$$
B_{1}=\bigcup_{i=1}^{3} P_{i}
$$

where $P_{1}, P_{2}$ and $P_{3}$ are paths, $V\left(P_{i}\right) \cap V\left(P_{j}\right)=\{a, b\}$ for $1 \leq i<j \leq 3$ and if $B$ is a block of $G$ with $B \neq B_{1}$ then $B$ is an edge.

Remark 3.1. The set $T=\{a, b\}$, where $a$ and $b$ are defined in (18), is a cutset. Assume from now on that the ideal $J(G)$ is unmixed. Let $l_{i}$ be the length of the path $P_{i}$ in (18) such that

$$
1 \leq l_{1} \leq l_{2} \leq l_{3} .
$$

If $l_{1}=1$, that is $\{a, b\} \in E(G)$, then $a$ is a cutpoint and $b$ is not a cutpoint. In fact by Lemma 1.2, $c(T)=3$. But we have exactly two connected components induced by the paths $P_{2}$ and $P_{3}$. Hence there exists another connected component in $G_{\bar{T}}$ that is not a subgraph of $B_{1}$ and the assertion follows. By a similar argument if $l_{1}>1$ then neither a nor $b$ is a cutpoint.

All over the section we use the notation defined in (18) and Remark 3.1. We call these graphs non-cactus bicyclic graphs.

Lemma 3.2. Let $G$ be a non-cactus bicyclic graph such that $J(G)$ is unmixed. We use notation (18). Then 
(1) each path $P_{i}$ has length less than 4 ;

(2) at most one path $P_{i}$ has length 3.

Proof. (1). Suppose by contradiction that exists a path $P \in\left\{P_{1}, P_{2}, P_{3}\right\}$ of length $l \geq 4$. Let $a, a_{1}, c, b_{1}$ and $b, 5$ distinct vertices of $V(P)$, with $\left\{a, a_{1}\right\},\left\{b, b_{1}\right\} \in E(P)$. Let $T=\{a, b\}$ and suppose that neither $a$ nor $b$ is a cutpoint. We observe that $T_{1}=\left\{a, b_{1}\right\}$ is a cutset. Hence $c\left(T_{1}\right)=3$. But in $G_{\bar{T}_{1}}$ there are two connected components that are subgraphs of $B_{1}$, one containing the vertex $c$ and one containing the vertex $b$. Hence there exists another block $B_{2}$ different from $B_{1}$ that contains the vertex $b_{1}$. Let $E\left(B_{2}\right)=\left\{\left\{b_{1}, b_{1}^{\prime}\right\}\right\}$. By the same argument there exists a block $B_{3}$ such that $E\left(B_{3}\right)=\left\{\left\{a_{1}, a_{1}^{\prime}\right\}\right\}$. The set $T_{2}=\left\{a_{1}, b_{1}\right\}$ is a cutset and induces 4 components in $G_{\bar{T}_{2}}$ : The first containing $a$ and $b$, the second containing $a_{1}^{\prime}$, the third containing $b_{1}^{\prime}$, and the fourth containing $c$. Contradiction. By similar argument is left to the reader to check that if $a$ cutpoint and $b$ is not a cutpoint, then $T_{1}=\{b, c\}$ is a cutset with $c$ a cutpoint. Hence we have a contradiction focusing on the cutset $\{a, c\}$.

(2). Suppose by contradiction that exist 2 paths $P_{1}$ and $P_{2}$ of length 3 such that

$$
V\left(P_{i}\right)=\left\{a, a_{i}, b_{i}, b\right\}, E\left(P_{i}\right)=\left\{\left\{a, a_{i}\right\},\left\{a_{i}, b_{i}\right\},\left\{b_{i}, b\right\}\right\} \text { for } i=1,2 .
$$

By Remark 3.1 we assume the vertex $b$ is not a cutpoint and we focus on the cutset $T=\left\{b, a_{1}\right\} . G_{\bar{T}}$ has two connected components that are subgraphs of $B_{1}$, one containing the vertex $a$ and one containing the isolated vertex $b_{1}$. Hence there exists another block $B_{2}$ different from $B_{1}$ that contains the vertex $a_{1}$. Let $E\left(B_{2}\right)=\left\{\left\{a_{1}, a_{1}^{\prime}\right\}\right\}$. By the same argument there exists a block $B_{3}$ such that $E\left(B_{3}\right)=\left\{\left\{a_{2}, a_{2}^{\prime}\right\}\right\}$. The set $T_{1}=\left\{b, a_{1}, a_{2}\right\}$ is a cutset with 5 components in $G_{\bar{T}_{1}}$ : the first containing $a$, the second (respectively the third) containing $a_{1}^{\prime}$ (respectively $a_{2}^{\prime}$ ) and the 2 isolated vertices $b_{1}$ and $b_{2}$. Contradiction.

Lemma 3.3. Let $G$ be a non-cactus bicyclic graph. Then $J(G)$ is CohenMacaulay (respectively unmixed but not Cohen-Macaulay) if and only if $B(G)$ is a tree and $G$ is decomposable into indecomposable graphs $G_{1}, \ldots, G_{r}$, and such that $G_{1}$ is one of the graphs in Figure 7 (respectively Figure 8).

Proof. Let $\left(l_{1}, l_{2}, l_{3}\right) \in \mathbb{N}^{3}$ where $l_{i}$ is the length of the paths $P_{i}$. By Remark 3.1 and Lemma 3.2 we have to study the following 4 cases:

$$
(1,2,2),(1,2,3),(2,2,2),(2,2,3) \text {. }
$$

Case $(1,2,2)$. Since $l_{1}=1$ we obtain the graph on the left in Figure 7 that is Cohen-Macaulay. In fact, if we consider the cone from the vertex $a$ to the two connected components given by the isolated vertex $f_{3}$ and the path whose edges are $\left\{f_{1}, b\right\}$ and $\left\{b, f_{2}\right\}$, by Theorem 3.8 of [9], we have the assertion. 

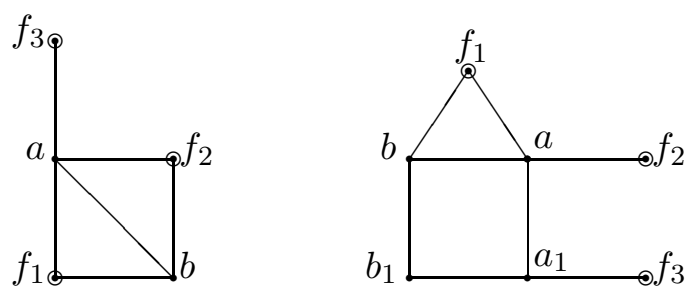

Figure 7. Bicyclic Cohen-Macaulay non-cactus graphs.

Case $(1,2,3)$. Since $l_{1}=1$ we obtain the graph on the right in figure 7 removing the vertex $f_{3}$. Using similar argument to the one used in Proposition 2.2, we obtain that the only candidate is exactly the graph in figure 7] and

$$
\mathcal{C}(G)=\left\{\emptyset,\{a\},\left\{a_{1}\right\},\left\{a, a_{1}\right\},\{a, b\},\left\{a, b_{1}\right\},\left\{a_{1}, b\right\},\left\{a, a_{1}, b\right\}\right\} .
$$

We focus on the cutpoint $a$. By Corollary 1.5 we obtain $J(G)=J\left(G_{\bar{a}}\right) \cap Q_{a}$, moreover $Q_{a}=\left(x_{a}, y_{a}\right)+J(P)$ where $J(P)$ is the binomial edge ideal of the path with

$$
\begin{gathered}
E(P)=\left\{\left\{f_{1}, b\right\},\left\{b, b_{1}\right\},\left\{b_{1}, a_{1}\right\},\left\{a_{1}, f_{3}\right\}\right\}, \\
\mathcal{C}(P)=\left\{\emptyset,\left\{a_{1}\right\},\{b\},\left\{b_{1}\right\},\left\{a_{1}, b\right\}\right\} .
\end{gathered}
$$

The ring $S / Q_{a}$ is Cohen-Macaulay of dimension 8 and it has two components: The path $P$ on 5 vertices and the isolated vertex $f_{2}$. We also observe that $J\left(G_{\bar{a}}\right)$ is the cone from the vertex $a_{1}$ to the vertex $f_{3}$ and the graph obtained attaching the edge $\left\{b_{1}, b\right\}$ to the complete graph whose vertices are $\left\{a, b, f_{1}, f_{2}\right\}$. Hence $J\left(G_{\bar{a}}\right)$ is Cohen-Macaulay of dimension 8, too. Moreover $Q_{a}+J\left(G_{\bar{a}}\right)$ is a binomial edge ideal that is equal to the previous cone removing the vertex $a$, hence it is Cohen-Macaulay of depth 7. By the Depth Lemma applied on the following exact sequence the assertion follows

$$
0 \longrightarrow S / J(G) \longrightarrow S / Q_{a} \oplus S / J\left(G_{\bar{a}}\right) \longrightarrow S /\left(Q_{a}+J\left(G_{\bar{a}}\right)\right) \longrightarrow 0 .
$$

Cases $(2,2,2)$ and $(2,2,3)$. These two cases are unmixed if and only if we add the edges as in figure 8. Moreover we already found them in [10, Example 3.2 and 3.3. In that paper by symbolic computation we observed that they are not Cohen-Macaulay. In [2] there is an argument for the non Cohen-Macaualyness of the bipartite one, the one on the right of figure 8 .

Now we are ready to give the main result of the section.

Corollary 3.4. Let $G$ be a bicyclic graph. Then $J(G)$ is Cohen-Macaulay if and only if $B(G)$ is a tree, $G$ is decomposable into indecomposable graphs $G_{1}, \ldots, G_{r}$, and such that one of the following cases occurs:

(1) $G_{1}$ and $G_{2}$ are in the set of unicyclic graphs in Figure 3:

(2) $G_{1}$ is one of the bicyclic cactus graphs in Figure 4:

(3) $G_{1}$ is one of the bicyclic non-cactus graphs in Figure 7 . 


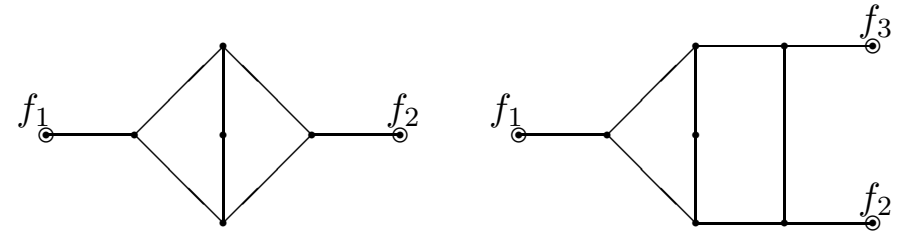

Figure 8. Bicyclic unmixed but non Cohen-Macaulay noncactus graphs.

\section{ACKNOWLEDGEMENTS}

The author was partially supported by GNSAGA of INdAM (Italy).

\section{REFERENCES}

[1] A. Banerjee, L. Nunez-Betancourt, Graph Connectivity and Binomial edge ideals, Proc. Amer. Math. Soc., Vol. 145 (2), 2017, pp. 487-499.

[2] D. Bolognini, A. Macchia, F. Strazzanti, Binomial edge ideals of bipartite graphs, arXiv:1704.00152 v1, 2017, pp. 1-29.

[3] CoCoATeam, CoCoA: a system for doing Computations in Commutative Algebra, Available at http://cocoa.dima.unige.it

[4] V. Ene, J. Herzog, T. Hibi, Cohen-Macaulay binomial edge ideals, Nagoya Math. J., Vol. 204, 2011, pp. 57-68.

[5] F. Harary, Graph theory, Addison-Wesley series in Mathematics, 1972.

[6] J. Herzog, T. Hibi, F. Hreinsdottir, T. Kahle, J. Rauh, Binomial edge ideals and conditional independence statements, Adv. in Appl. Math., Vol. 45, 2010, pp. 317333.

[7] D. Kiani, S. Saeedi Madani, Some Cohen-Macaulay and Unmixed Binomial Edge Ideals, Comm. Algebra, Vol. 43, 2015, pp. 5434-5453.

[8] M. Ohtani, Graphs and ideals generated by some 2-minors, Comm. Algebra, Vol. 39, 2011, pp. 905-917.

[9] A. Rauf, G. Rinaldo, Construction of Cohen-Macaulay binomial edge ideals, Comm. Algebra., Vol. 42, 2014, pp. 238-252.

[10] G. Rinaldo, Cohen-Macaulay binomial edge ideals of small deviation, Bull. Math. Soc. Sci. Math. Roumanie, Tome 56(104) No. 4, 2013, 497-503.

[11] R.H. Villarreal, Cohen-Macaulay graphs, Manuscripta Math., Vol. 66, 1990, pp. 277293.

Department of Mathematics, University of Trento, via Sommarive, 14, 38123 Povo (TRENTO), ITALY 